\title{
Narcissists Are Easy, Stepmothers Are Hard
}

\author{
Daniel Gorín ${ }^{1}$ and Lutz Schröder ${ }^{1,2}$ \\ 1 DFKI GmbH, Bremen, Germany \\ 2 Department of Computer Science, Universität Bremen, Germany \\ firstname.lastname@dfki.de
}

\begin{abstract}
Modal languages are well-known for their robust decidability and relatively low complexity. However, as soon as one adds a selfreferencing construct, like hybrid logic's down-arrow binder, to the basic modal language, decidability is lost, even if one restricts binding to a single variable. Here, we concentrate on the latter case and investigate the logics obtained by restricting the nesting depth of modalities between binding and use. In particular, for distances strictly below 3 we obtain well-behaved logics with a relatively high descriptive power. We investigate the fragment with distance 1 in the framework of coalgebraic modal logic, for which we provide very general decidability and complexity results. For the fragment with distance 2 we focus on the case of Kripke semantics and obtain optimum complexity bounds (no harder than the base logic). We show that this fragment is expressive enough to accommodate the guarded fragment over the correspondence language.
\end{abstract}

\section{Introduction}

Modal logics are known for their robust decidability and relatively low complexity. However, they don't play along well with binding constructs such as the $\downarrow$ binder of hybrid logic, which allows naming the current point of evaluation for later reference. Hybrid logic with $\downarrow$ and the satisfaction operator @ is a conservative reduction class for first-order logic and, therefore, has undecidable satisfiability and finite satisfiability problems (see, e.g., [3]). Undecidability in the presence of $\downarrow$ is rather robust. E.g. it persists without @, without nominals, and even if only one variable is allowed to be bound by $\downarrow$ (or, semantically, if only one state can be remembered at any given time) [14]. Also, satisfiability with respect to classes of models that are typically computationally well-behaved (e.g., linear, transitive or equivalence relation frames) is undecidable (except in the uni-modal case) [18. Weakened versions of $\downarrow$ were investigated and also turned out to be undecidable 2 .

Syntactic fragments of the hybrid language with $\downarrow$ were investigated in 22 . There, it is observed that undecidability of the logic with $\downarrow$ can be established by reduction from the tiling problem using a formula that contains the so-called $\square \downarrow \square$ pattern, i.e. a $\square$-modality occurs under the scope of a $\downarrow$ that occurs under the scope of a $\square$. It is then shown that interdicting this pattern (in negation normal forms) ensures decidability. 
Here, we investigate a different form of restriction, coming from the same observation. The reduction of the tiling problem given in [22] uses three modalities: $\square$ is made a master modality using a spypoint; $\diamond_{1}$ and $\nabla_{2}$ are forced to be total functions; and the crucial grid is defined by the formula

$$
\square \downarrow x \cdot \square\left(s \rightarrow \square\left(\diamond_{1} \diamond_{2} x \rightarrow \diamond_{2} \diamond_{1} x\right)\right) .
$$

Observe that there are four modalities between the binding of $x$ and its uses. Informally, we say that $x$ occurs at depth 4 from its binding. This raises the question of the decidability status of fragments of the logic with $\downarrow$ (and only one variable) where every use of the variable occurs at a given maximal depth $k$ from its binding. Known undecidabiltiy proofs work for $k=4$ [14]; as a first step in our investigation, we show that undecidability holds also for $k=3$.

We then show (Section 3) that for depth at most 1, and not only for the Kripke semantics of modal logic but for any logic in the framework of coalgebraic logic (which supports, e.g., probabilities, counting, preferences, and game logics) [1621, we obtain a decidable logic with the exponential model property. even when the global modality $A$ is added to the language. Under mild assumptions, it can be shown that satisfiability is in fact in EXPTIME, a tight bound in most cases. These results can be shown to hold also in the presence of nominals.

For Kripke semantics, we can improve these results in several ways (Section 4): we can allow up to depth 2 and retain decidability in EXPTIME, even though the finite model property breaks. Moreover, we establish a quasi-tree model property for the fragment without $A$ and prove decidability in PSPACE.

The language with $A$ and a depth bound of 2 for occurrence of the bound variable is quite expressive. In particular, we show (Section [5) that it subsumes the guarded fragment over the modal correspondence language (without constants and on formulas with two free variables). Because the guarded fragment does enjoy the finite model property, the containment is proper.

\section{Coalgebraic Logics with Self-reference}

For greater generality, we work in the framework of coalgebraic modal logic [16, which covers a broad range of modalities beyond the standard relational setup, including probabilistic and game-theoretic phenomena as well as neighbourhood semantics and non-material conditionals [21. This framework is parametric in syntax and semantics. The syntax is given by a similarity type $\Lambda$, i.e. a set of modal operators with finite arities $\geq 0$ (hence possibly including propositional atoms); to simplify notation, we will pretend that all operators are unary. Adopting the notation of [14], we use the personal pronouns I and me as binder and bindee, respectively. The full language $\mathcal{F}(\Lambda, \mathrm{I}, @, \mathrm{~A})$ is given by the grammar

$$
\mathcal{F}(\Lambda, \mathrm{I}, @, \mathrm{~A}) \ni \phi, \psi::=\perp \mid \text { me }|\phi \rightarrow \psi| \nabla \phi|\mathrm{A} \phi| \mathrm{I} . \phi \mid @_{\mathrm{me}} \phi
$$

where $\varnothing \in \Lambda$. We use the standard derived boolean operators $\neg$, $\wedge$, etc., while $E$ denotes the dual of $A$. Occasionally, we will assume all formulas to be in 
negation normal form (nnf) and in such cases $\neg, \wedge$ and $\vee$ will be taken as primitive. We will also usually restrict our attention to syntactic fragments of $\mathcal{F}(\Lambda, \mathrm{I}, @, \mathrm{~A})$, such as $\mathcal{F}(\Lambda, \mathrm{I}, @)$ or $\mathcal{F}(\Lambda, \mathrm{I})$ : the fragments without $\mathrm{A}$ and with neither A nor @, respectively. We use $\operatorname{rank}(\phi)$ to denote the maximum number of nested occurrences of $\varnothing \in \Lambda$ in $\phi$. The notion of rank is trivially extended to finite sets of formulas.

The semantics of the logic is given by an endofunctor $T:$ Set $\rightarrow$ Set and,

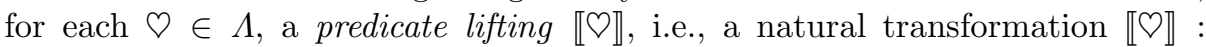
$\mathcal{Q} \rightarrow \mathcal{Q} \circ T^{o p}$, where $\mathcal{Q}$ is the contravariant powerset functor Set $^{o p} \rightarrow$ Set (i.e. $\mathcal{Q} X=2^{X}$ and $\left.\mathcal{Q} f(A)=f^{-1}[A]\right)$. As usual, $T$ is assumed w.l.o.g. to preserve injective maps [5] and to be non-trivial, in the sense that $T X=\emptyset$ implies $X=\emptyset$.

Models of the logic are $T$-coalgebras $\langle X, \gamma\rangle$, where $X$ is a non-empty set of states and $\gamma: X \rightarrow T X$ is the transition function. Given two states $x, y \in X$ (the current and the remembered state, respectively), the truth value of $\mathcal{F}(\Lambda, \mathrm{I}, @$ @, A)formulas is inductively defined by:

$$
\begin{array}{ll}
y, x \models_{\gamma} \text { me } \Longleftrightarrow x=y & y, x \models_{\gamma} \mathrm{A} \phi \Longleftrightarrow \forall z \in X . z, z \\
y, x \models_{\gamma} \nabla \phi \Longleftrightarrow \gamma(x) \in \llbracket \nabla \rrbracket_{X} \llbracket \phi \rrbracket_{\gamma, y} & y, x \models_{\gamma} @_{\text {me }} \phi \Longleftrightarrow y, y \models_{\gamma} \phi \\
y, x \models_{\gamma} \mathrm{I} . \phi \Longleftrightarrow x, x \models_{\gamma} \phi &
\end{array}
$$

where $\llbracket \phi \rrbracket_{\gamma, x}=\left\{z \in X \mid x, z \models_{\gamma} \phi\right\}$; Boolean operations were omitted. When clear from context, we shall write simply $y, x \models \phi$ and $\llbracket \phi \rrbracket_{x}$.

Occurrences of me in $\phi$ that are not under the scope of an I-binder are said to be free. A formula that contains no free occurrences of me is called a sentence.

Lemma 1. If $\phi$ is a sentence, then $y, x \models_{\gamma} \phi$ iff $x, x \models_{\gamma} \phi$, for all $x, y$.

Therefore, for a sentence $\phi$, we may omit the remembered state and write $x=_{\gamma} \phi$.

Example 2. 1. Kripke semantics is an instance of coalgebraic semantics: For $n<\omega$, the signature $K_{n}$ has unary modal operators $\square_{1}, \square_{2}, \ldots \square_{n}$ and a countably infinite set $\mathrm{P}$ of propositional atoms (nullary operators). As usual, $\diamond_{i}$ is the dual of $\square_{i}$, i.e. $\vee_{i} \phi=\neg \square_{i} \neg \phi$. The semantics is given by the endofunctor $T$ defined by $T X=(\mathcal{P} X)^{n} \times \mathcal{P}$ P, equipped with predicate liftings $\llbracket \square_{i} \rrbracket_{X}(C)=$ $\left\{\left(A_{1}, \ldots, A_{n}, B\right) \in T X \mid A_{i} \subseteq C\right\}$ and $\llbracket p \rrbracket_{X}=\left\{\left(A_{1}, \ldots, A_{n}, B\right) \in T X \mid p \in B\right\}$ for $p \in \mathrm{P}$. Then $T$-coalgebras assign to each state an $n$-tuple of sets of successors and a valuation for $\mathrm{P}$, and hence are exactly Kripke models with $n$ relations. We shall denote these as $\mathcal{P}^{n}$-models $\langle X, \gamma, \pi\rangle$ with $\gamma: X \rightarrow(\mathcal{P} X)^{n}$ and $\pi: X \rightarrow \mathcal{P P}$.

2. In graded logic [8] one has modal operators $\nabla_{k}$ for $k \geq 0$, read 'in more than $k$ successors, it holds that'. We can interpret this logic over the functor $\mathcal{B}$ that takes a set $X$ to the set $\mathcal{B} X$ of multisets over $X$, i.e. maps $B: X \rightarrow \mathbb{N} \cup\{\infty\}$, using the predicate liftings $\llbracket \diamond_{k} \rrbracket_{X}(A)=\left\{B \in \mathcal{B}(X) \mid \sum_{x \in A} B(x)>k\right\}$. This captures the multigraph semantics of graded modalities [7, which in the absence of I-me engenders the same notion of satisfiability as the more standard Kripke semantics of graded modalities [19]. 
3. Probabilistic logic [13] has operators $L_{p}$ 'in the next step, it holds with probability at least $p$, for $p \in[0,1] \cap \mathbb{Q}$. Its semantics is based on the functor $\mathcal{D}$ mapping $X$ to the set of discrete probability distributions on $X$, with $\llbracket L_{p} \rrbracket_{X}(A)=\{P \in \mathcal{D}(X) \mid P A \geq p\} . \mathcal{D}$-coalgebras are Markov chains.

Other modal logics that fit in the coalgebraic paradigm include neighbourhood semantics, coalition logic, and non-monotonic conditionals $\Rightarrow$ ("if - then normally'), to name just a few [21].

Remark 3. One may consider variants $@_{\text {me }}^{*}$ and $A^{*}$ of the operators $@_{\text {me }}$ and $A$ with semantic clauses $y, x \models_{\gamma} @_{\text {me }}^{*} \phi \Longleftrightarrow x, y \models_{\gamma} \phi$ and $y, x \models_{\delta}^{\tau} \mathrm{A}^{*} \phi \Longleftrightarrow$ $\forall z \in X . y, z \models_{\delta}^{\tau} \phi$, respectively (so that $@_{\text {me }}=@_{\text {me }}^{*}$ I and $\mathrm{A}=\mathrm{A}^{*} \mathrm{I}$ ). The operator $@_{\text {me }}^{*}$ is not in general expresible in $\mathcal{F}(\Lambda, \mathrm{I}, @, \mathrm{~A})$ : one can show using the notion of bisimulation introduced in Section 4 that the formula I. $\diamond\left(\neg\right.$ me $\wedge @_{\text {me }}^{*} \diamond \neg$ me $)$ is not expressible in $\mathcal{F}\left(K_{n}, \mathrm{I}, @\right.$, A $)$. In the relational case, one can however give a polynomial reduction using inverse relations (which can be defined in $\mathcal{F}\left(K_{n}, \mathrm{I}\right.$, @ ) as shown below). Also $\mathrm{A}^{*}$ is not in general expressible in $\mathcal{F}(\Lambda, \mathrm{I}, @, \mathrm{~A})$ : in the relational case, a sink can be defined by the formula I. $A^{*}(\neg$ me $\rightarrow \diamond$ me); again, inexpressibility of this property in $\mathcal{F}\left(K_{n}, \mathrm{I}\right.$, @, A) is shown using bisimulations. Technically, $A^{*}$ has a number of undesirable properties; e.g. unlike A it cannot be reduced to TBox reasoning (Remark 4), and moreover it subverts the semantic idea behind the depth restriction.

Remark 4. In the following, we will work with TBoxes in the style of description logic instead of the A operator in full generality, i.e. we will assume that all formulas are of the form $(\mathrm{A} \phi) \wedge \psi$ where $\phi, \psi$ do not contain A; satisfiability of such a formula will be referred to as satisfiability of $\psi$ over (the TBox) $\phi$. Similarly, we refer to validity over $\phi$, soundness over $\phi$ etc. Algorithmically, we can reduce the A-operator to TBox reasoning as follows. We first guess which subformulas $A \phi$ of the target formula are valid (one can do this already in NP; algorithms for A happen in EXPTIME). Given this guess, we can eliminate all occurrences of $A$, thus ending up with a modified target formula, a TBox, and a conjunction $\neg \mathrm{A} \rho_{1} \wedge \cdots \wedge \neg \mathrm{A} \rho_{k}$. As our logic is invariant under coproducts of models, it now suffices to check (separately) that the target formula and the formulas $\neg \rho_{i}$ are satisfiable over the TBox.

We are interested in the modal distance between every bound occurrence of me and its associated I. We now make this notion precise. We define sets of formulas $\mathcal{F}_{i}^{n}(\Lambda, \mathrm{I}, @, \mathrm{~A})(0 \leq i \leq n)$ intuitively containing those formulas where i) every free occurrence of me is under at most $i-1$ modalities; and ii) there are at most $n$ modalities between every bound me and its corresponding I:

$$
\begin{aligned}
& \mathcal{F}_{0}^{n}(\Lambda, \mathrm{I}, @, \mathrm{~A}) \ni \phi_{0}^{n}, \psi_{0}^{n}::=\quad \perp|p| \phi_{0}^{n} \rightarrow \psi_{0}^{n}\left|\nabla \phi_{0}^{n}\right| \mathrm{I} . \phi_{n}^{n} \mid \mathrm{A} \phi_{0}^{n} \\
& \mathcal{F}_{l}^{n}(\Lambda, \mathrm{I}, @, \mathrm{~A}) \ni \phi_{l}^{n}, \psi_{l}^{n}::=\mathrm{me}|\perp| p\left|\phi_{l}^{n} \rightarrow \psi_{l}^{n}\right| \nabla \phi_{l-1}^{n}\left|\mathrm{I} . \phi_{n}^{n}\right| \mathrm{A} \phi_{0}^{n} \mid @_{\mathrm{me}} \phi_{l}^{n}
\end{aligned}
$$

where $l>0$. Notice that me does not occur in $\mathcal{F}_{0}^{n}(\Lambda, \mathrm{I}, @, \mathrm{~A})$. According to the grammar, me cannot occur free under $A$, which is not a real restriction as any 
Table 1. Encoding of the grid of an $\mathbb{N} \times \mathbb{N}$ tiling in $\mathcal{F}_{3}\left(K_{9}, \mathrm{I}\right)$. Here $L=\{u, r, u r, r u\}$.

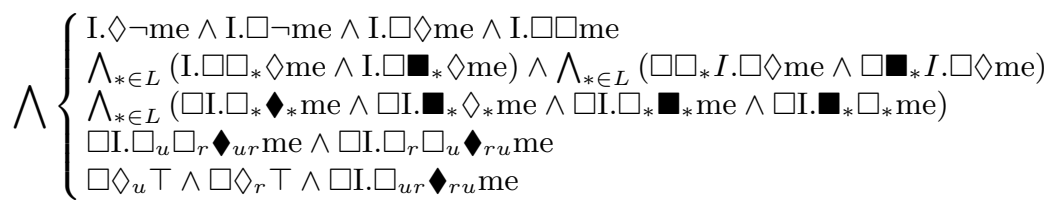

formula $\mathrm{A} \phi$ is equivalent to AI. $\phi$. We will usually write $\mathcal{F}_{n}(\ldots)$ instead of $\mathcal{F}_{n}^{n}(\ldots)$ and informally refer to it as the formulas of depth $n$, which form the depth-n fragment. We assume w.l.o.g. that I occurs only in front of modal operators.

Theorem 5. Satisfiability is undecidable for $\mathcal{F}_{4}\left(K_{1}, \mathrm{I}\right)$ and for $\mathcal{F}_{3}\left(K_{9}, \mathrm{I}\right)$.

Proof (sketch). Both claims are shown by reduction from the $\mathbb{N} \times \mathbb{N}$ tiling problem (cf. [6]) using the so-called spypoint technique. The proof for $\mathcal{F}_{4}\left(K_{1}, \mathrm{I}\right)$ can be found in [14] (a simpler one but for $\mathcal{F}_{4}\left(K_{3}, \mathrm{I}\right)$ is given in [22]). Here we show the construction for $\mathcal{F}_{3}\left(K_{9}, \mathrm{I}\right)$. For the sake of clarity, we assume $K_{9}=\left\{\square, \square_{u}, \square_{r}, \square_{u r}, \square_{r u}, \mathbf{\square}_{u}, \mathbf{\square}_{r}, \mathbf{\square}_{u r}, \mathbf{\square}_{r u}\right\} ; \square_{u}$ and $\square_{r}$ represent moving one step, up or right, in the grid, while $\square_{u r}$ and $\square_{r u}$ correspond to a two-step move up-right and right-up. Each $\boldsymbol{\square}_{*}$ is intended to be the inverse of $\square_{*}$. We use $\square$ to go back and forth between the points of the grid and the spypoint. The latter is the initial point of evaluation. The encoding of the grid is shown in Table 1. The first two lines define a spypoint. The next line makes $\square_{*}$ and $\boldsymbol{\sigma}_{*}$ inverses and, exploiting this fact, injective functions. The last two lines make $\square_{u r}$ and $\square_{r u}$ composites of $\square_{u}$ and $\square_{r}$, and force $\square_{u}$ and $\square_{r}$ to commute, respectively.

The stepmother example from [14] (I. $\rangle_{\text {hasSpouse }} \diamond_{\text {has Child }} \square_{\text {hasParent }} \neg$ me) lives in the depth-3 fragment, while the celebrity needs only depth 2 (I. $\left.\square_{\text {meets }} \diamond_{\text {knows }} \mathrm{me}\right)$. Although it is possible to reduce the number of relation symbols required in the proof above (see, e.g. [14]), the decidability of $\mathcal{F}_{3}\left(K_{1}, \mathrm{I}\right)$ remains open.

\section{The Depth-1 Fragment in Coalgebraic Semantics}

As a first step in our program, we investigate the depth-1 fragment, which allows for defining the narcissist ( $I . \diamond_{\text {loves }}$ me) and the egotist $\left(I . \square_{\text {loves }} . m e\right)$, but can also be applied in the coalgebraic setting to express phenomena such as usually knowing oneself on Tuesdays (if maybe not on Mondays), $I$. Tuesday $\Rightarrow_{\text {knows }}$ me (here we index conditionals by role names) or a Markov chain staying in the current state with probability at least $2 / 3\left(I . L_{2 / 3} \mathrm{me}\right)$. Specifically, we prove that even at the general coalgebraic level, the depth-1 fragment has the exponential model property over general TBoxes (and hence in the presence of $A$ ), with ensuing generic decidability and complexity results.

As we work in the depth-1 fragment, we can assume that every occurrence of $\varnothing \in \Lambda$ is prefixed by I, and we regard I. $\odot$ as a single operator - consequently, 
the subformulas of I. $\nabla \phi$ are I. $\nabla \phi$ and the subformulas of $\phi$, but not $\nabla \phi$. We next recall some notation and previous results.

Definition 6. We use $\operatorname{Prop}(V)$ to denote the set of Boolean expressions over a set $V$ (of atoms) and put $\Lambda(V)=\{$ I. $\varnothing v \mid \varnothing \in \Lambda, v \in V\}$. For any set $X$, an $X$-substitution for $V$ is a function $V \rightarrow X$. Given $\alpha \in \operatorname{Prop}(V)$ and a $\mathcal{P}(X)$ substitution $\tau, \llbracket \alpha \rrbracket_{X, \tau}$ is the extension of $\alpha$ in the boolean algebra $\mathcal{P}(X)$ under the interpretation $\tau$. Similarly, for a functor $T$ and predicate liftings $\llbracket \nabla \rrbracket$, we write $\llbracket \alpha \rrbracket_{T X, \tau}$, with $\alpha \in \operatorname{Prop}(\Lambda(V))$ and $\tau$ a $\mathcal{P}(X)$-substitution for $V$, to denote the extension of $\alpha$ in the boolean algebra $\mathcal{P}(T X)$ under the interpretation function $\lambda \oslash v$. $₫ \rrbracket_{X}(\tau(v))$. We say that that $\alpha$ is one-step satisfiable over $\tau$ if $\llbracket \alpha \rrbracket_{T X, \tau} \neq \emptyset$.

Definition 7. A clause over $V$ is a disjunction of atoms in $V \cup \neg V$. A onestep rule over a set $V$ is a pair $\phi / \psi$ where $\phi \in \operatorname{Prop}(V)$ and $\psi$ is a clause over $\Lambda(V)$. A one-step rule $\phi / \psi$ is one-step sound if whenever $\llbracket \phi \rrbracket_{X, \tau}=X$, then $\llbracket \psi \rrbracket_{T X, \tau}=T X$. A collection $\mathcal{R}$ of one-step rules is one-step complete if whenever $\llbracket \chi \rrbracket_{T X, \tau}=T X$ with $\chi$ a clause over $\Lambda(V)$, then there exist $\phi / \psi \in \mathcal{R}$ over $V$ and a $V$-substitution $\sigma$ such that $\psi \sigma$ propositionally entails $\chi$ and $\llbracket \phi \sigma \rrbracket_{X, \tau}=X$.

In proofs, a one-step rule $\phi / \psi$ over $V$ is applied in substituted form, i.e. conclude $\psi \sigma$ from $\phi \sigma$, where $\sigma$ substitutes formulas for the variables in $V$. Thanks to the following result, we can assume that we have a one-step complete set $\mathcal{R}$ of onestep sound rules.

Theorem 8 ([19]). The set of all one-step sound rules for a functor $T$ and its corresponding predicate liftings is one-step complete.

We will first show that whenever $\phi$ is satisfiable over a TBox $\chi$, then $\phi$ has an exponentially bounded model (in $|\chi|+|\phi|$ ) satisfying $\chi$. To this end, we let $\Sigma$ be the closure of $\{\phi, \chi\}$ under subformulas (in the above sense), negation, and prefixing with @ me, where we identify $\neg \neg \phi$ with $\phi, \neg @_{\text {me }} \phi$ with @ me $\neg$, and $@_{\text {me }} @_{\text {me }} \phi$ with $@_{\text {me }} \phi$. We use $\Sigma^{\prime}$ to denote the set of sentences in $\Sigma$. We let $S_{\chi}$ be the set of all maximally satisfiable subsets of $\Sigma^{\prime}$ containing $\chi$.

We build a syntactic model whose domain $2 S_{\chi}$ contains two copies of each $B \in S_{\chi}$. Formally, we define $2 S_{\chi}=\{\top, \perp\} \times S_{\chi}$ and let $l: 2 S_{\chi} \rightarrow S_{\chi}$ denote the second projection. For $A \in S_{\chi}$, let $S_{\chi, A}$ denote the set of maximally satisfiable subsets of $\Sigma$ (which may contain free occurrences of me) extending $\{\chi\} \cup @_{\mathrm{me}} A$, where $@_{\mathrm{me}} A=\left\{@_{\mathrm{me}} \psi \mid \psi \in A\right\}$. For each $(a, A) \in 2 S_{\chi}$, we define a function $r_{(a, A)}: S_{\chi, A} \rightarrow 2 S_{\chi}$ as $r_{(a, A)}(B)=\left(b, B \cap \Sigma^{\prime}\right)$ where $b=\top$ iff $B$ entails the formula $a \leftrightarrow$ me.

For $x \in 2 S_{\chi}$, we define a valuation $\tau_{x}: V_{\Sigma} \rightarrow \mathcal{P}\left(2 S_{\chi}\right)$ on $V_{\Sigma}=\left\{a_{\rho} \mid \rho \in \Sigma\right\}$ by $\tau_{x}\left(a_{\rho}\right)=[\rho]_{x}$, with $[\rho]_{x}$ given by the usual clauses for Boolean operators plus

$$
\begin{aligned}
{[a]_{x} } & =\left\{y \in 2 S_{\chi} \mid a \in l(y)\right\} & {[\mathrm{me}]_{x} } & =\{x\} \\
{\left[@_{\operatorname{me}} \rho\right]_{x} } & =\left\{y \in 2 S_{\chi} \mid x \in[\rho]_{x}\right\} & {[\mathrm{I} . \nabla \rho]_{x} } & =\left\{y \in 2 S_{\chi} \mid \text { I. } . \rho \rho \in l(y)\right\} .
\end{aligned}
$$

Also, define $\eta_{A}: V_{\Sigma} \rightarrow \mathcal{P}\left(S_{\chi, A}\right)$ by $\eta_{A}\left(a_{\rho}\right)=\left\{B \in S_{\chi, A} \mid \rho \in B\right\}$. The following key lemma, whose proof depends crucially on the fact that for every $x \in 2 S_{\chi}$, $r_{x}: S_{\chi l(x)} \rightarrow 2 S_{\chi}$ is injective, allows us to move from the complex definition of $\tau_{x}$ to the simpler $\eta_{l(x)}$ : 
Lemma 9. For all $\rho \in \Sigma$ and $x \in 2 S_{\chi}, \eta_{l(x)}\left(a_{\rho}\right)=r_{x}^{-1}\left[\tau_{x}\left(a_{\rho}\right)\right]$.

We let $\sigma_{\Sigma}: V_{\Sigma} \rightarrow \Sigma$ by $\sigma_{\Sigma}\left(a_{\rho}\right)=\rho$ and then have, similar to Lemma 27 in [19],

Lemma 10. For $A \in S_{\chi}$ and $\theta \in \operatorname{Prop}\left(V_{\Sigma}\right)$, $\| \theta \rrbracket_{S_{\chi, A}, \eta_{A}}=S_{\chi, A}$ iff $@_{\operatorname{me}} \wedge A \rightarrow$ $\theta \sigma_{\Sigma}$ is valid over the TBox $\chi$ ( $\bigwedge A$ is the conjunction of all formulas in $A$ ).

Lemma 11 (Existence). There is a coherent coalgebra structure $\xi$ on $2 S_{\chi}$, i.e., one such that $\xi(x) \in \llbracket \nabla \rrbracket[\rho]_{x}$ iff $x \in[\mathrm{I} . \nabla \rho]_{x}$ for every $x$ and every $\mathrm{I} . \nabla \rho \in \Sigma$.

The key point in the proof of the existence lemma in comparison to the base case [19] is the following lemma.

Lemma 12 (Rule relativization). If $\theta / \psi$ is a sound one-step rule, then we can soundly conclude $\rho \rightarrow \psi \sigma$ from $@_{\text {me }} \rho \rightarrow \theta \sigma$, for any substitution $\sigma$ and any sentence $\rho$.

Lemma 13 (Truth). Let $\xi$ be a coherent coalgebra structure on $2 S_{\chi}$. Then $x, y \models_{\xi} \rho$ iff $y \in[\rho]_{x}$ for $\rho \in \Sigma$ and hence $x, y=_{\xi} \rho^{\prime}$ iff $\rho^{\prime} \in l(y)$ for $\rho^{\prime} \in \Sigma^{\prime}$.

Theorem 14. $\mathcal{F}_{1}(\Lambda, \mathrm{I}, @, \mathrm{~A})$ has the exponential model property. Moreover, if one-step satisfiability is in NP, satisfiability is NEXPTIME; when the former is in $P$, the latter is in EXPTIME.

Proof. The exponential model property is established by the above construction, as $2 S_{\chi}$ has exponential size. One-step satisfiability in NP allows guessing and checking an exponential model in exponential time [19]. With one-step satisfiability in $\mathrm{P}$ we can use Hintikka set elimination, similarly as in [20].

Remark 15. The EXPTIME bound is tight already in the relational case. Almost all instances of coalgebraic logics, including conditional logics, alternating-time/coalition logics, and probabilistic logics, have one-step satisfiability problems in $\mathrm{P}$ [19, so that the EXPTIME result applies in these cases. We have little doubt that using global caching, one can show an EXPTIME bound for socalled EXPTIME-tractable instances [9], which would cover essentially all cases of interest. For graded logics (whose one-step satisfiability problem is NP-complete), we have recent results proving an EXPTIME bound even at depth 2 [10].

Remark 16. Theorem 14 generalizes to a setting with nominals [3], i.e. propositional symbols $i$ denoting single states which can appear by themselves or in satisfaction operators $@_{i} \rho$ (' $\rho$ holds at state $i$ '). That is, the result also holds in the framework of coalgebraic hybrid logic [15]. Only minor modifications of the construction are required, the most notable one being that elements of $S_{\chi}$ that are named, i.e. contain a positive nominal, are not duplicated in the construction of the carrier. This is in sharp contrast to the depth- 2 case, studied next, where nominals cause drastic effects even in the relational case (Remark 25). 


\section{The Depth-2 Fragment in Kripke Semantics}

We will next we focus on the depth-2 fragment under relational semantics, i.e., $\mathcal{F}_{2}\left(K_{n}, \mathrm{I}, @\right.$, A $)$ and some of its fragments. Syntactically, we view this as a logic with two types of modal operators, I. $\square_{i}$ and $\square_{i}$; by the depth- 2 restriction, no $\square_{i}$ can occur directly under another $\square_{j}$. Our main result is decidability in EXPTIME (even in PSPACE without A). This contrasts with Theorem 5 and, more generally, with the robust undecidability of $\mathcal{F}\left(K_{n}, \mathrm{I}\right)$ (Section 1).

A detailed observation of the proof of Theorem 5 shows that $\mathcal{F}_{2}\left(K_{n}, \mathrm{I}, @\right.$ @, A) is quite expressive: only two formulas are needed outside this fragment. In particular, Table 11llustrates that in depth-2, one can define inverse and functional relations, and similarly one can define reflexive and symmetric relations. As another example, consider the formula $\gamma=\diamond \top \wedge \mathrm{I} . \square(\neg$ me $\wedge \diamond \mathrm{me}) \wedge \square \mathrm{I} . \square \diamond \neg \mathrm{me}$. It is not hard to see that $x \models \gamma$ implies that $x$ has at least two successors, i.e. satisfies the formula $\diamond^{>1} T$, where $\diamond^{>1}$ is a graded modality (cf. Example 22). We can delineate the expressive power of $\mathcal{F}_{2}\left(K_{n}, \mathrm{I}\right.$, @, A) using a notion of bisimulation:

Definition 17. Let $\langle X, \gamma, \pi\rangle$ and $\langle Y, \delta, \tau\rangle$ be $\mathcal{P}^{n}$-models. Then $Z_{1}, Z_{2} \subseteq X^{2} \times$ $Y^{2}$ constitute an $I @ \mathrm{~A}_{2}$-bisimulation whenever they satisfy all the conditions in Table 2. The weaker notions of $\mathrm{I}_{2^{-}}, \mathrm{IA}_{2^{-}}$and $\mathrm{I}_{2}$-bisimulations are obtained by ignoring clauses $\left\{A_{l}, A_{r}\right\},\{@\}$ and $\left\{A_{l}, A_{r}, @\right\}$, respectively.

Proposition 18. Let $\langle X, \gamma, \pi\rangle$ and $\langle Y, \delta, \tau\rangle$ be two $\mathcal{P}^{n}$-models such that relations $Z_{1}, Z_{2} \subseteq X^{2} \times Y^{2}$ constitute an $\mathrm{I} @ \mathrm{~A}_{2}$-bisimulation between them. Then:

1. $\left(x_{0}, x_{1}\right) Z_{1}\left(y_{0}, y_{1}\right) \Longrightarrow\left(x_{0}, x_{1} \models_{\gamma}^{\pi} \phi \Longleftrightarrow y_{0}, y_{1} \models_{\delta}^{\tau} \phi\right) \forall \phi \in \mathcal{F}_{1}\left(K_{n}, \mathrm{I}, @\right.$ @ , A $)$

2. $\left(x_{0}, x_{1}\right) Z_{2}\left(y_{0}, y_{1}\right) \Longrightarrow\left(x_{0}, x_{1} \models_{\gamma}^{\pi} \phi \Longleftrightarrow v_{0}, v_{1} \models_{\delta}^{\tau} \phi\right) \forall \phi \in \mathcal{F}_{2}\left(K_{n}, \mathrm{I}, @, \mathrm{~A}\right)$

Analogous results hold for $\mathrm{I}_{2^{-}}, \mathrm{IA}_{2}-$ and $\mathrm{I}_{2}$-bisimulations.

Example 19. Formula $\gamma$ above shows that $\mathcal{F}_{2}\left(K_{n}, \mathrm{I}\right)$ can express some cardinality requirements on successors. We make this expressivity more precise by showing that it cannot distinguish structures with two and three successors. Consider two $\mathcal{P}$-models $\langle X, \gamma, \pi\rangle$ and $\langle A, \delta, \tau\rangle$ where $X=\left\{x, y_{0}, y_{1}\right\} ; A=$ $\left\{a, b_{0}, b_{1}, b_{2}\right\} ; \pi(p)=\tau(p)=\emptyset$ for all proposition $p$; and $\gamma$ and $\delta$ are such that

Table 2. Conditions that define a IA@ $@_{2}$-bisimulation. $Z_{*}$ stands for both $Z_{1}$ and $Z_{2}$.

(p) $\quad\left(x_{0}, x_{1}\right) Z_{*}\left(y_{0}, y_{1}\right) \Longrightarrow\left(x_{1} \in \pi(p) \Longleftrightarrow y_{1} \in \tau(p)\right)$, for each proposition $p$

(me) $\left(x_{0}, x_{1}\right) Z_{*}\left(y_{0}, y_{1}\right) \Longrightarrow\left(x_{0}=x_{1} \Longleftrightarrow y_{0}=y_{1}\right)$

(I. $\left.\square_{l}\right)\left(x_{0}, x_{1}\right) Z_{1}\left(y_{0}, y_{1}\right)$ and $x_{1} R_{i} x_{2} \Longrightarrow \exists y_{2}$ st. $y_{1} R_{i} y_{2}$ and $\left(x_{1}, x_{2}\right) Z_{*}\left(y_{1}, y_{2}\right)$

$\left(\square_{l}\right)\left(x_{0}, x_{1}\right) Z_{2}\left(y_{0}, y_{1}\right)$ and $x_{1} R_{i} x_{2} \Longrightarrow \exists y_{2}$ st. $y_{1} R_{i} y_{2}$ and $\left(x_{0}, x_{2}\right) Z_{1}\left(y_{0}, y_{2}\right)$

(I. $\left.\square_{r}\right)\left(x_{0}, x_{1}\right) Z_{1}\left(y_{0}, y_{1}\right)$ and $y_{1} R_{i} y_{2} \Longrightarrow \exists x_{2}$ st. $x_{1} R_{i} x_{2}$ and $\left(x_{1}, x_{2}\right) Z_{*}\left(y_{1}, y_{2}\right)$

$\left(\square_{r}\right)\left(x_{0}, x_{1}\right) Z_{2}\left(y_{0}, y_{1}\right)$ and $y_{1} R_{i} y_{2} \Longrightarrow \exists w_{2}$ st. $x_{1} R_{i} x_{2}$ and $\left(x_{0}, x_{2}\right) Z_{1}\left(y_{0}, y_{2}\right)$

(@) $\left(x_{0}, x_{1}\right) Z_{*}\left(y_{0}, y_{0}\right) \Longrightarrow\left(x_{0}, x_{0}\right) Z_{*}\left(y_{0}, y_{0}\right)$

( $\left.\mathrm{A}_{l}\right) \quad \forall x \exists y$ st. $(x, x) Z_{*}(y, y)$

$\left(\mathrm{A}_{r}\right) \forall y \exists x$ st. $(x, x) Z_{*}(y, y)$ 
$R_{\gamma}=\left\{\left(x, y_{i}\right) \mid 0 \leq i \leq 1\right\}$ and $R_{\delta}=\left\{\left(a, b_{i}\right) \mid 0 \leq i \leq 2\right\}$. It is not hard to verify that $Z_{1}=Z_{2}=\left\{(s, t) \in X^{2} \times A^{2} \mid \forall 0 \leq i \leq 1, \pi_{i}(s)=a \Longleftrightarrow \pi_{i}(t)=x\right\}$ constitute an $\mathrm{I}_{2}$-bisimulation.

\section{$4.1 \mathcal{F}_{2}\left(K_{n}, I\right)$ Is PSPACE-Complete}

Using Proposition[18 we will show that $\mathcal{F}_{2}\left(K_{n}, \mathrm{I}\right)$ possesses a variant of the treemodel property: every satisfiable formula has a model that is contained in the reflexive-symmetric closure of a tree. Moreover these quasi-tree-models can be shown to be shallow, i.e., have a depth polynomial on the size of the formula. As usual, this is key in deriving a decision procedure for satisfiability that runs in polynomial space.

Definition 20. We say that $S \subseteq X^{2}$ is a quasi-tree with root $r$ if for some tree $R$ with root $r, R \subseteq S \subseteq R \cup R^{-1} \cup \operatorname{Id}_{X}$. A $\mathcal{P}^{n}$ model $\langle X, \gamma, \pi\rangle$ is a quasi-tree-model with root $r$ when $\bigcup_{i} R_{\gamma, i}$ is a quasi-tree with root $r$.

Theorem 21 (Quasi-tree model property). If $\phi \in \mathcal{F}_{2}\left(K_{n}, I\right)$ is a satisfiable sentence then there exists a quasi-tree model that satisfies $\phi$ at its root.

Proof (sketch). By Lemma 1 let $\mathcal{X}$ be a model with domain $X$ such that $r, r \models_{\gamma}^{\pi}$ $\phi$, for some $r \in X$. One builds an unravelling $\mathcal{A}$ of $\mathcal{X}$ such that it contains all the paths in $\mathcal{X}$ from $r$ where no self-loop is ever taken and such that one never immediately returns to the preceding state, regardless of the relation used. This unravelling gives us a quasi-tree and one then shows it to be bisimilar $\mathcal{X}$.

We are now ready to introduce the announced tableau construction. In this context, we will restrict our attention to $\mathcal{F}_{2}\left(K_{n}, \mathrm{I}\right)$-formulas in nnf subject to the following restriction: if me occurs free in $\phi$, then it does so under the scope of some modality. Notice that for such a formula $\square_{i} \phi, \phi$ need not satisfy this condition. We solve this by defining, for an arbitrary formula $\phi, \phi(\top)$ and $\phi(\perp)$ as the result of replacing every free occurrence of me that is not under a modality by $\top$ and $\perp$, respectively. E.g., for $\phi=$ me $\wedge \square_{i}$ me, $\phi(\perp)=\perp \wedge \square_{i}$ me; the former does not satisfy the restriction above, the latter does.

Given a set of formulas $\Sigma$, its closure $C l(\Sigma)$, is the smallest set that is closed under nnf negation and pseudo-subformulas, that is, if $\phi \in C l(\Sigma)$ and $\psi$ is a subformula of $\phi$, then $\psi(\top)$ and $\psi(\perp)$ are in $C l(\Sigma)$ as well. $C l^{*}(\Sigma)$ denotes the set of sentences in $C l(\Sigma)$.

Let $\phi$ be a $\mathcal{F}_{2}\left(K_{n}, \mathrm{I}\right)$-sentence. A tableau for $\phi$ is then a labelled quasi-tree $\mathcal{T}=\left\langle T, R,{ }^{\mathcal{T}}\right\rangle$ with root $r$, where each directed edge $(s, t) \in R$ is labelled with a non-empty set of relation indices $(s, t)^{\mathcal{T}} \subseteq\{1 \ldots n\}$ (we write $s R_{i} t$ if $(s, t) \in \mathcal{T}$ and $\left.i \in(s, t)^{\mathcal{T}}\right)$ and each node is labelled with a tuple $s^{\mathcal{T}}=\left\langle H_{*}, H_{* *}, H_{f_{*}}, H_{* f}\right\rangle$ where $H_{*}$ is a Hintikka set over (some subset of) $C l^{*}(\{\phi\})$ and all the others are Hintikka sets over (subsets of) $C l(\{\phi\})$ (for an $s \in T$, we will denote these sets by $s_{*}^{\mathcal{T}}, s_{* *}^{\mathcal{T}}, s_{f *}^{\mathcal{T}}$ and $s_{* f}^{\mathcal{T}}$; or even $s_{*}, s_{* *}, s_{f *}$ and $s_{* f}$ if $\mathcal{T}$ is clear from context).

Intuitively, $s_{*}^{\mathcal{T}}$ contains sentences that hold at $s$ (where the remembered state is irrelevant); $s_{* *}^{\mathcal{T}}$ contains formulas that hold at $s$ when the remembered state is 
Table 3. Conditions for a tableau $\mathcal{T}$ for $\phi$ with root $r$. For $s \in T$, fs is the father of $s$ (if $s \neq r$ ); and $t_{1}, \ldots t_{k} \in T$ are the children of $s$. We use $t$ to designate some existentially quantified children of $s$. Also $L=\{*, * *, f *, * f\}$.

1. $\phi \in r_{*}$ and $r_{f *}=r_{* f}=\emptyset$.

2. $\bigcup_{x \in L} s_{x} \subseteq C l(\{\phi\})$ and $\operatorname{rank}\left(\bigcup_{x \in L} s_{x}\right)>\operatorname{rank}\left(\bigcup_{x \in L} t_{j_{x}}\right)$ for all $j$.

3. For a sentence $\psi$, if $\psi \in s_{* *} \cup s_{f *}$ or $\psi \in t_{* f}$, then $\psi \in s_{*}$.

4. If $\nabla_{i} \psi \in s_{*}$ or I. $\nabla_{i} \psi \in s_{*}$, then some of the following must hold:
i) $s R_{i} f s, \psi(\perp) \in s_{* f}$; ii) $s R_{i} s, \psi(\top) \in s_{* *}$; iii) $s R_{i} t, \psi(\perp) \in t f *$.

5. If $\square_{i} \psi \in s_{*}$ or I. $\square_{i} \psi \in s_{*}$, then all of the following must hold:

i) $s R_{i} f s \Longrightarrow \psi(\perp) \in s_{* f}$; ii) $s R_{i} s \Longrightarrow \psi(\top) \in s_{* *}$; iii) $s R_{i} t \Longrightarrow \psi(\perp) \in t_{f *}$.

6. If $\diamond_{i} \psi \in s_{* *}$ (with me free in $\psi$ ), then some of the following must hold:

i) $s R_{i} f s, \psi(\perp) \in f s_{*}$; ii) $s R_{i} s, \psi(\top) \in s_{*}$; iii) $s R_{i} t, \psi(\perp) \in t_{*}$.

7. If $\square_{i} \psi \in s_{* *}$ (with me free in $\psi$ ), then all of the following must hold:

i) $s R_{i} f s \Longrightarrow \psi(\perp) \in f s_{*}$; ii) $s R_{i} s \Longrightarrow \psi(\top) \in s_{*}$; iii) $s R_{i} t \Longrightarrow \psi(\perp) \in t_{*}$.

8. If $\diamond_{i} \psi \in s_{f *}$ (with me free in $\psi$ ), then some of the following must hold:

i) $s R_{i} f s, \psi(\top) \in f s_{*}$; ii) $s R_{i} s, \psi(\perp) \in s_{*}$; iii) $s R_{i} t, \psi(\perp) \in t_{*}$.

9. If $\square_{i} \psi \in s_{f *}$ (with me free in $\psi$ ), then all of the following must hold:

i) $s R_{i} f s \Longrightarrow \psi(\top) \in f s_{*}$; ii) $s R_{i} s \Longrightarrow \psi(\perp) \in s_{*}$; iii) $s R_{i} t \Longrightarrow \psi(\perp) \in t_{*}$.

10. If $\diamond_{i} \psi \in t_{j_{* f}}$ (with me free in $\psi$ ), then some of the following must hold:

i) $s R_{i} f s, \psi(\perp) \in f s_{*}$; ii) $s R_{i} s, \psi(\perp) \in s_{*}$;

iii) $s R_{i} t_{j}, \psi(\top) \in t_{j_{*}}$; iv) $s R_{i} t_{k}, j \neq k, \psi(\perp) \in t_{k_{*}}$.

11. If $\square_{i} \psi \in t_{j_{* f}}$ (with me free in $\psi$ ), then all of the following must hold:

i) $s R_{i} f s \Longrightarrow \psi(\perp) \in f s_{*}$; ii) $s R_{i} s \Longrightarrow \psi(\perp) \in s_{*}$;

iii) $s R_{i} t_{j} \Longrightarrow \psi(\top) \in t_{j_{*}}$; iv) $s R_{i} t_{k}$ with $j \neq k \Longrightarrow \psi(\perp) \in t_{k *}$.

$s, s_{f *}^{\mathcal{T}}$ the ones that hold when the remembered state is the father of $s$ and $s_{* f}^{\mathcal{T}}$ the formulas that hold at the father of $s$ when the remembered state is $s$. This intuition suggests the conditions in Table 3. Notice that conditions 7 [11 heavily use the fact that, because I is assumed to occur always in front of a modality, me cannot occur free under a modality in $\psi$.

Lemma 22. There exists a tableau for a sentence $\phi$ iff $\phi$ is satisfiable.

Lemma 23. Let $\phi$ be a satisfiable sentence. Every tableau for $\phi$ has depth bounded by rank $(\phi)$. Moreover, $\phi$ has a tableau with breadth polynomial in $|\phi|$.

Theorem 24. The satisfiability problem for $\mathcal{F}_{2}\left(K_{n}, \mathrm{I}\right)$ is PSPACE-complete.

Remark 25. Unlike the depth-1 case (Remark 16) this result does not hold in the presence of nominals, were we can form a spypoint using the formula: $s \wedge \square\left(\diamond s \wedge \bigwedge_{i} \square_{i} \mathrm{I} . \diamond(s \wedge \diamond \mathrm{me})\right)$, internalizing global reasoning. This gives us the possibility of defining functions and inverses, which in combination with nominals cause NEXPTIME-hardness and break the finite model property.

\section{$4.2 \mathcal{F}_{2}\left(K_{n}, \mathrm{I}, @\right)$ Is PSPACE-Complete}

It is not hard to see that $\mathcal{F}_{2}\left(K_{n}, \mathrm{I}\right.$, @ $)$ is as expressive as $\mathcal{F}_{2}\left(K_{n}, \mathrm{I}\right)$, although perhaps more succinctly so. Indeed, as an example, consider the form 
$\square_{i} \mathrm{I} \cdot \square_{j}\left(p \vee \square_{k}\left(@_{\mathrm{me}} \diamond_{l}\right.\right.$ me $\left.\left.\rightarrow p\right)\right)$. A trivial transformation leads to the equivalent formula $\square_{i} \mathrm{I}$. $\left(\left(\diamond_{l}\right.\right.$ me $\left.\wedge \square_{j}\left(p \vee \square_{k}(\top \rightarrow p)\right)\right) \vee\left(\neg \diamond_{l}\right.$ me $\left.\left.\wedge \square_{j}\left(p \vee \square_{k}(\perp \rightarrow p)\right)\right)\right)$. It is not hard to generalize this example into a truth-preserving translation from $\mathcal{F}_{2}\left(K_{n}, \mathrm{I}, @\right)$ to $\mathcal{F}_{2}\left(K_{n}, \mathrm{I}\right)$ but with an exponential blow-up in size.

Lemma 26. There exists a polynomial, satisfiability preserving translation from $\mathcal{F}_{2}\left(K_{n}, \mathrm{I}, @\right)$ to $\mathcal{F}_{2}\left(K_{n}, \mathrm{I}\right)$.

Proof. We use the standard technique of replacing subformulas by fresh propositional symbols. We illustrate the idea translating the example used above and leave the formal details to the reader. The translated formula we obtain in this case is $\square_{i} \mathrm{I}$. $\left(\diamond_{l}\right.$ me $\left.\leftrightarrow q \wedge\left(q \rightarrow \square_{j} \square_{k} q\right) \wedge\left(\neg q \rightarrow \square_{j} \square_{k} \neg q\right) \wedge \square_{j}\left(p \vee \square_{k}(q \rightarrow p)\right)\right)$, where $q$ is fresh. Notice that formulas $(\neg) q \rightarrow \square_{j} \square_{k}(\neg) q$ are used to propagate the value of $q$ to the modal context in which the @-formula originally occurred.

Theorem 27. Satisfiability for $\mathcal{F}_{2}\left(K_{n}, \mathrm{I}\right.$, @) is PSPACE-complete.

\section{3 $\mathcal{F}_{2}\left(K_{n}, I\right.$, @, A) Is EXPTIME-Complete}

We begin by observing that the proof of Lemma 26 can be used to show also that $\mathcal{F}_{2}\left(K_{n}, \mathrm{I}\right.$, @, A $)$ is polynomially reducible to $\mathcal{F}_{2}\left(K_{n}, \mathrm{I}, \mathrm{A}\right)$, so the latter is the logic we will consider. Moreover, we reduce A to TBoxes (Remark 4).

Now, it is not hard to adapt conditions 2 in Table 3 in order to obtain a tableau for $\mathcal{F}_{2}\left(K_{n}, \mathrm{I}, \mathrm{A}\right)$ such that an analogue of Lemma 22 holds. However, we will not be able to give a bound for the depth of this tableau. In fact, since $\mathcal{F}_{2}\left(K_{n}, \mathrm{I}, \mathrm{A}\right)$ encodes inverse functional roles, standard examples show

Theorem 28. $\mathcal{F}_{2}\left(K_{2}, \mathrm{I}, \mathrm{A}\right)$ lacks the finite model property.

We will show that we can nevertheless effectively decide existence of infinite

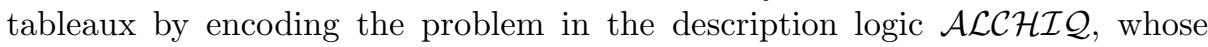
satisfiability problem with respect to general TBoxes is EXPTIME-complete [12. As a modal language (i.e. avoiding the more common DL syntax), $\mathcal{A L C H \mathcal { C }}$ contains graded modalities $\diamond_{i}^{>n}$ and $\square_{i}^{\leq n}$ and inverse modalities (denoted by $\boldsymbol{\nabla}_{i}$ and $\boldsymbol{\square}_{i}$ ). Additionally it features role inclusion axioms $R_{i} \sqsubseteq R_{j}$, which roughly correspond to AI. $\square_{i} \boldsymbol{}_{j}$ me. For an introduction to description logics, see [4].

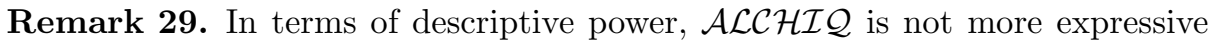
than $\mathcal{F}_{2}\left(K_{n}, \mathrm{I}\right)$; for instance $\square$ I. $\diamond$ me has no $\mathcal{A L C H \mathcal { I } Q}$ equivalent.

Assume we are given a $\mathcal{F}_{2}\left(K_{n}, \mathrm{I}\right)$-formula $\phi$ and a general TBox $\chi$. The encoding into $\mathcal{A L C H \mathcal { I }}$ will use relation symbols $R_{1}, \ldots, R_{n}$ and $R_{f}$, with role inclusion axioms $R_{i}^{-1} \sqsubseteq R_{f}$ for $1 \leq i \leq n$ (where $R_{i}^{-1}$ denotes the inverse relation of $R_{i}$ ). Moreover, we impose a TBox axiom $\square_{f}^{\leq 1} \top$, so that $R_{f}$ is interpreted as a partial function. This essentially means that we work with tree-models, where $R_{f}$ represents the "father-of" relation. Notice that a node may reach its children by more than one $R_{i}$. Since models will be proper trees, we will need to make explicit provisions for quasi-trees in the encoding. 
Table 4. Encoding of conditions 311 in Table 3 as global axioms in $\mathcal{A L C H \mathcal { Q }}$

3. $\bigwedge_{\psi \in \Sigma_{0}}\left(\rho_{* *: \psi} \rightarrow \rho_{*: \psi}\right) \wedge\left(\rho_{f *: \psi} \rightarrow \rho_{*: \psi}\right) \wedge\left(\rho_{* f: \psi} \rightarrow \diamond_{f} \rho_{*: \psi}\right)$

4. $\bigwedge_{(\mathrm{I} .) \diamond_{i} \psi \in \Sigma_{0}} \rho_{*:(\mathrm{I} .) \diamond_{i} \psi} \rightarrow\left(\left(\uparrow_{i} \wedge \rho_{* f: \psi(\perp)}\right) \vee\left(\circlearrowleft_{i} \wedge \rho_{* *: \psi(\mathrm{T})}\right) \vee \diamond_{i} \rho_{f *: \psi(\perp)}\right)$

5. $\bigwedge_{(\mathrm{I} .) \square_{i} \psi \in \Sigma_{0}} \rho_{*:(\mathrm{I} .) \square_{i} \psi} \rightarrow\left(\left(\uparrow_{i} \rightarrow \rho_{* f: \psi(\perp)}\right) \wedge\left(\circlearrowleft_{i} \rightarrow \rho_{* *: \psi(\mathrm{T})}\right) \wedge \square_{i} \rho_{f *: \psi(\perp)}\right)$

6. $\bigwedge_{\diamond_{i} \psi \in \Sigma_{1}} \rho_{* *: \diamond_{i} \psi} \rightarrow\left(\left(\uparrow_{i} \wedge \square_{f} \rho_{*: \psi(\perp)}\right) \vee\left(\circlearrowleft_{i} \wedge \rho_{*: \psi(\top)}\right) \vee \diamond_{i} \rho_{*: \psi(\perp)}\right)$

7. $\bigwedge_{\square_{i} \psi \in \Sigma_{1}} \rho_{* *: \square_{i} \psi} \rightarrow\left(\left(\uparrow_{i} \rightarrow \square_{f} \rho_{*: \psi(\perp)}\right) \wedge\left(\circlearrowleft_{i} \rightarrow \rho_{*: \psi(\mathrm{T})}\right) \wedge \square_{i} \rho_{*: \psi(\perp)}\right)$

8. $\bigwedge_{\diamond_{i} \psi \in \Sigma_{1}} \rho_{f *: \diamond_{i} \psi} \rightarrow\left(\left(\uparrow_{i} \wedge \square_{f} \rho_{*: \psi(T)}\right) \vee\left(\circlearrowleft_{i} \wedge \rho_{*: \psi(\perp)}\right) \vee \diamond_{i} \rho_{*: \psi(\perp)}\right)$

9. $\bigwedge_{\square_{i} \psi \in \Sigma_{1}} \rho_{f *: \square_{i} \psi} \rightarrow\left(\left(\uparrow_{i} \rightarrow \square_{f} \rho_{*: \psi(\top)}\right) \wedge\left(\circlearrowleft_{i} \rightarrow \rho_{*: \psi(\perp)}\right) \wedge \square_{i} \rho_{*: \psi(\perp)}\right)$

10. $\bigwedge_{\diamond_{i} \psi \in \Sigma_{1}} \rho_{* f: \diamond_{i} \psi} \rightarrow\left(\begin{array}{l}\diamond_{f}\left(\uparrow_{i} \wedge \rho_{*: \psi(\perp)}\right) \vee \diamond_{f}\left(\circlearrowleft_{i} \wedge \rho_{*: \psi(\perp)}\right) \vee\left(\diamond_{i} \top \wedge \rho_{*: \psi(\top)}\right) \\ \vee\left(\rho_{*: \neg \psi(\perp)} \wedge \diamond_{f} \diamond_{i} \rho_{*: \psi(\perp)}\right) \vee\left(\rho_{*: \psi(\perp)} \wedge \diamond_{f} \diamond_{i}^{>1} \rho_{*: \psi(\perp)}\right)\end{array}\right)$

11. $\wedge_{\square_{i} \psi \in \Sigma_{1}} \rho_{* f: \square_{i} \psi} \rightarrow\left(\begin{array}{l}\vartheta_{f}\left(\uparrow_{i} \rightarrow \square_{f} \rho_{*: \psi(\perp)}\right) \wedge \diamond_{f}\left(\circlearrowleft_{i} \rightarrow \rho_{*: \psi(\perp)}\right) \\ \wedge\left(\mathbf{\square}_{i} \perp \diamond_{f} \square_{i} \rho_{*: \psi(\perp)}\right) \\ \wedge \diamond_{i} \top \rightarrow\left(\rho_{*: \psi(\top)} \wedge\left(\begin{array}{l}\rho_{*: \psi(\perp)} \rightarrow \diamond_{f} \square_{i} \rho_{*: \psi(\perp)} \wedge \\ \rho_{*: \neg \psi(\perp)} \rightarrow \diamond_{f} \square_{i}^{\leq 1} \rho_{*: \psi(\perp)}\end{array}\right)\right)\end{array}\right)$

Let $L=\{*, * *, f *, * f\}, \Sigma=C l(\{\chi, \psi\}), \Sigma_{0}=C l^{*}(\{\chi, \psi\})$ and $\Sigma_{1}=\Sigma \backslash \Sigma_{0}$. We use the set $V=\left\{\rho_{l: \psi} \mid l \in L, \psi \in \Sigma\right\} \cup\left\{\circlearrowleft_{i} \mid 1 \leq i \leq n\right\} \cup\left\{\uparrow_{i} \mid 1 \leq i \leq n\right\}$ of proposition symbols. Intuitively we want $\rho_{l: \psi}$ to hold at a state $s$ of a (quasi-)tree if $\psi \in s_{l}$. Moreover $\circlearrowleft_{i}$ and $\uparrow_{i}$ are used to denote that state $s$ has a self-loop or reaches his father, respectively.

Since we want $\chi$ to hold globally, we impose the TBox axiom $\rho_{*: \chi}$. Also, we require nodes to be labelled with Hintikka sets by means of the TBox axiom $\bigwedge_{l \in L}\left(\neg \rho_{l: \perp} \wedge \bigwedge_{\psi \in \Sigma} \neg\left(\rho_{l: \psi} \wedge \rho_{l: \neg \psi}\right) \wedge \bigwedge_{\psi_{1} \star \psi_{2} \in \Sigma}\left(\rho_{l: \psi_{1} \star \psi_{2}} \rightarrow \rho_{l: \psi_{1}} \star \rho_{l: \psi_{2}}\right)\right)$.

To ensure a correct distinction between the root and non-root nodes, we impose TBox axioms $\uparrow_{i} \rightarrow \nabla_{f} \top$ which state that $\uparrow_{i}$ can only hold at non-root nodes. Moreover, conditions 3 to 11 are encoded by the TBox axioms shown in Table 4. The encoding is straightforward, except for conditions 10 and 11, since $\mathcal{A L C H \mathcal { I }}$ provides no direct way of expressing the requirement $t_{j} \neq t_{k}$ (cf. Table 3). In the case of condition 10. for instance, we overcome this by splitting into cases: if $t_{j} \not \models \psi(\perp)$, then any $t_{k}$ that satisfies $\psi(\perp)$ will be different from $t_{i}$; otherwise, we use the graded modality $\diamond^{>1}$ to ensure another one exists.

Existence of a tableau for $A \chi \wedge \phi$ then amounts to the satisfiability, over the TBox described above, of the formula $\rho_{* \phi} \wedge \square_{f} \perp \wedge \bigwedge_{\psi \in \Sigma_{0}}\left(\neg \rho_{f *: \psi} \wedge \neg \rho_{* f: \psi}\right)$.

Theorem 30. Satisfiability for $\mathcal{F}_{2}\left(K_{n}, \mathrm{I}, @\right.$ @, A) is EXPTIME-complete.

\section{Embedding the Guarded Fragment}

Modal correspondence theory studies the relation between modal logic and classical logics, most notably, first-order logic (cf. [23]). The link is typically established through the modal correspondence language: a first-order language with only one-place and two-place relation symbols; the former stand for proposition 
symbols on the modal side, the latter for (relational) modalities. Kripke models can then be seen as relational models, and well-known translations allow embedding many modal logics into first-order logic (FO).

The guarded fragment of FO [1, characterized by its guarded quantification pattern, generalizes the modal fragment of FO. It enjoys good computational properties: both the finite model property and a form of tree-model property, and an EXPTIME-complete satisfiability problem (under bounded arity) [1].

We will see that the guarded fragment over the modal correspondence language can be almost perfectly (i.e. using fresh relation symbols whose interpretation is uniquely determined by that of the old symbols) embedded into $\mathcal{F}_{2}\left(K_{n}\right.$, I, @, A). While they agree on the complexity of satisfiability, because the latter lacks the finite model property (Theorem 28), the containment is proper.

We need to make precise what we mean by an almost perfect embedding. For $K_{n}=\left\{\square_{1}, \ldots, \square_{n}\right\}$, let tense $\mathcal{F}_{2}\left(K_{n}, \mathrm{I}, @\right.$, A $)$ to be the logic $\mathcal{F}_{2}\left(K_{2 n}, \mathrm{I}, @\right.$, A $)$, with $K_{2 n}=\left\{\square_{1}, \ldots \square_{n}, \mathbf{a}_{1}, \ldots \mathbf{\square}_{n}\right\}$, restricted to the class of models that satisfy the formula $A \bigwedge_{i \leq n}\left(\mathrm{I} . \square_{i} \boldsymbol{\diamond}_{i}\right.$ me $\wedge$ I. $\boldsymbol{\square}_{i} \searrow_{i}$ me), i.e. those where $\square_{i}$ and $\boldsymbol{\square}_{i}$ are interpreted as inverse relations. There is a bijection between models for $\mathcal{F}_{2}\left(K_{n}, \mathrm{I}, @\right.$ @ , A) and models for tense $\mathcal{F}_{2}\left(K_{n}, \mathrm{I}, @, \mathrm{~A}\right)$.

Theorem 31. Given a guarded formula $\alpha\left(x_{1}, x_{2}\right)$ in the correspondence language for $K_{n}$, there is a $\phi$ in tense $\mathcal{F}_{2}\left(K_{n}, \mathrm{I}, @, \mathrm{~A}\right)$ such that for every $\mathcal{C}, \mathcal{C} \models_{\text {fo }}$ $\alpha\left[x_{1} \mapsto c_{1}, x_{2} \mapsto c_{2}\right]$ iff $c_{1}, c_{2} \models \phi$.

Proof. First, observe that every guarded formula with two free variables over the correspondence language is equivalent to a guarded formula of FO2. Moreover, the set of guarded formulas of FO2 can be split into four overlapping sets $G^{\emptyset}$, $G^{x_{1}}, G^{x_{2}}$ and $G^{x_{1} x_{2}}$, such that $\alpha \in G^{\star}$ iff the free variables of $\alpha$ are among $\star$. These sets can be described by simple, mutually recursive grammars, as shown in Table 5, where $x$ ranges over $\left\{x_{1}, x_{2}\right\}, \dot{x_{1}}=x_{2}$ and $\dot{x_{2}}=x_{1}$. Finally, Table 6 exhibits translation functions (Booleans omitted) for each of these sets. An induction over $\alpha \in G^{\star}$ shows that $\mathcal{C} \models_{\text {fo }} \alpha\left[\dot{x} \mapsto c_{1}, x \mapsto c_{2}\right]$ iff $c_{1}, c_{2} \models T_{\dot{x} x}^{\star}(\alpha)$.

Remark 32. One point to observe about Table 6 is that modal operators appear without a preceding I only in formulas of the form $\diamond_{i}$ me and $\boldsymbol{}_{i}$ me; contrastingly, $\mathcal{F}_{2}\left(K_{n}, \mathrm{I}\right)$ would also allow formulas such as $\diamond_{i}(\neg$ me $\wedge \phi)$. Indeed one can show that the guarded fragment is essentially the extension of $\mathcal{F}_{1}\left(K_{n}, \mathrm{I}\right.$, @, A) with

Table 5. The guarded fragment of FO2 is the union of $G^{\emptyset}, G^{x_{1}}, G^{x_{2}}$ and $G^{x_{1}, x_{2}}$

\begin{tabular}{lll}
\multicolumn{1}{c}{$G^{\emptyset} \ni \alpha, \beta$} & \multicolumn{1}{c}{$G^{x} \ni \alpha(x), \beta(x)$} & $G^{x \dot{x}} \ni \alpha(x, \dot{x}), \beta(x, \dot{x})$ \\
\hline$\neg \alpha \mid \alpha \vee \beta$ & $x=x|P x| R x x \mid \alpha$ & $x=\dot{x}|R x \dot{x}| R \dot{x} x$ \\
$\exists x . x=x \wedge \alpha(x)$ & $\neg \alpha(x) \mid \alpha(x) \vee \beta(x)$ & $\alpha|\alpha(x)| \alpha(\dot{x})$ \\
$\exists x . P x \wedge \alpha(x)$ & $\exists \dot{x} \cdot x=\dot{x} \wedge \alpha(x, \dot{x})$ & $\neg \alpha(x, \dot{x})$ \\
$\exists x . R x x \wedge \alpha(x)$ & $\exists \dot{x} \cdot R x \dot{x} \wedge \alpha(x, \dot{x})$ & $\alpha(x, \dot{x}) \vee \beta(x, \dot{x})$ \\
$\exists x \exists \dot{x} . R x \dot{x} \wedge \alpha(x, \dot{x})$ & $\exists \dot{x} \cdot R \dot{x} x \wedge \alpha(x, \dot{x})$ &
\end{tabular}


Table 6. Translations from the guarded fragment of FO2 to tense $\mathcal{F}_{2}\left(K_{n}, \mathrm{I}, @\right.$, A)

$$
\begin{aligned}
T_{\dot{x} x}^{x}(x=x) & =\top \\
T_{\dot{x} x}^{x}(P x) & =p \\
T_{\dot{x} x}^{x}\left(R_{i} x x\right) & =\mathrm{I} \cdot \diamond_{i} \mathrm{me} \\
T_{\dot{x} x}^{x}(\alpha) & =T_{\dot{x} x}^{\emptyset}(\alpha), \text { if } \alpha \in G^{\emptyset} \\
T_{\dot{x} x}^{x}(\exists \dot{x} \cdot x=\dot{x} \wedge \alpha) & =\mathrm{I} \cdot T_{\dot{x} x}^{x_{1} x_{2}}(\alpha) \\
\left.T_{\dot{x} x}^{x}\left(\exists \dot{x} \cdot R_{i} x \dot{x} \wedge \alpha\right)\right) & =\mathrm{I} \cdot \diamond_{i} T_{x \dot{x}}^{x_{1} x_{2}}(\alpha) \\
T_{\dot{x} x}^{x}\left(\exists \dot{x} \cdot R_{i} \dot{x} x \wedge \alpha\right) & =\mathrm{I} \cdot \diamond_{i} T_{x \dot{x}}^{x_{1} x_{2}}(\alpha) \\
T_{\dot{x} x}^{\emptyset}(\exists x \cdot x=x \wedge \alpha) & =\mathrm{EI} \cdot T_{\dot{x} x}^{x}(\alpha) \\
T_{\dot{x} x}^{\emptyset}(\exists x \cdot P x \wedge \alpha) & =\mathrm{EI} \cdot\left(p \wedge T_{\dot{x} x}^{x}(\alpha)\right) \\
T_{\dot{x} x}^{\emptyset}\left(\exists x \cdot R_{i} x x \wedge \alpha\right) & =\mathrm{E}\left(\mathrm{I} \cdot \diamond_{i} \operatorname{me} \wedge \mathrm{I} \cdot T_{\dot{x} x}^{x}(\alpha)\right) \\
T_{\dot{x} x}^{\emptyset}\left(\exists x \exists \dot{x} \cdot R_{i} x \dot{x} \wedge \alpha\right) & =\mathrm{EI} \cdot \diamond_{i} T_{x \dot{x}}^{x_{1} x_{2}}(\alpha)
\end{aligned}
$$

$$
\begin{aligned}
& T_{\dot{x} x}^{\dot{x}}(\dot{x}=\dot{x})=\top \\
& T_{\dot{x} x}^{\dot{x}}(P \dot{x})=@_{\mathrm{me}} p \\
& T_{\dot{x} x}^{\dot{x}}\left(R_{i} \dot{x} \dot{x}\right)=@_{\mathrm{me}} \mathrm{I} \cdot \diamond_{i} \mathrm{me} \\
& T_{\dot{x} x}^{\dot{x}}(\alpha)=T_{\dot{x} x}^{\emptyset}(\alpha), \text { if } \alpha \in G^{\emptyset} \\
& T_{\dot{x} x}^{\dot{x}}(\exists x . x=\dot{x} \wedge \alpha)=@_{\mathrm{me}} T_{\dot{x} x}^{x_{1} x_{2}}(\alpha) \\
& T_{\dot{x} x}^{\dot{x}}\left(\exists x \cdot R_{i} \dot{x} x \wedge \alpha\right)=@_{\mathrm{me}} \mathrm{I} \cdot \diamond_{i} T_{\dot{x} x}^{x_{1} x_{2}}(\alpha) \\
& T_{\dot{x} x}^{\dot{x}}\left(\exists x \cdot R_{i} x \dot{x} \wedge \alpha\right)=@_{\mathrm{me}} \mathrm{I} \cdot \boldsymbol{}_{i} T_{\dot{x} x}^{x_{1} x_{2}}(\alpha) \\
& T_{\dot{x} x}^{x_{1} x_{2}}(x=\dot{x})=\text { me } \\
& T_{\dot{x} x}^{x_{1} x_{2}}\left(R_{i} \dot{x} x\right)=\searrow_{i} \text { me } \\
& T_{\dot{x} x}^{x_{1} x_{2}}\left(R_{i} x \dot{x}\right)=\diamond_{i} \text { me } \\
& T_{\dot{x} x}^{x_{1} x_{2}}(\alpha)=T_{\dot{x} x}^{*}(\alpha)\left(\alpha \in G^{*}\right)
\end{aligned}
$$

$\diamond_{i}$ me (noting that also the definition of inverses as in Table 1 needs only $\diamond_{i}$ me). Thus, one might call the guarded fragment the 'depth-1.5' fragment — use of me is unrestricted at depth 1 , and limited to positive occurrences at depth 2 when $\diamond$ is taken as the basic modal operator. In fact, formulas of the form $\diamond_{i} \neg$ me can be encoded in the guarded 2-variable fragment with counting quantifiers (which is also known to be in EXPTIME [17]), while it does not seem easily possible to encode formulas of the more general form $\diamond_{i}(\neg \mathrm{me} \wedge \phi)$.

\section{Conclusion}

Modal logics extended with the I-me operators (or alternatively, $\downarrow$ with only one variable) are robustly undecidable. In this paper we have identified decidable fragments, based on the modal distance (depth) between the binder I and the bound variable me. We have shown that already for depth 3 the logic becomes undecidable (previous undecidability proofs required depth 4). However, for depth less than 3 we obtain well-behaved logics with a relatively high descriptive power. Indeed, for depth 2 we arrive at a logic that is EXPTIME-complete and strictly more expressive than the (constant-free) guarded fragment of the correspondence language. When restricted to local satisfiability (i.e., without TBoxes nor a universal modality), the problem was shown to be PSPACE-complete.

For depth 1 we obtained a very general result: coalgebraic modal logics extended in this way have an exponential model property, even with general TBoxes and nominals. Generalizing our results for depth 2 to the coalgebraic case is the subject of ongoing work; recent results [10] show that they do extend to the graded case. Unlike for depth 1, nominals cannot be added to the depth-2 logic without losing most of the good properties even in the relational case: even one nominal makes the logic NEXPTIME-hard and causes infinite branching. We conjecture that the depth-2 logic with nominals is NEXPTIME-complete. 


\section{References}

1. Andréka, H., van Benthem, J., Németi, I.: Modal languages and bounded fragments of predicate logic. J. Phil. Log. 27(3), 217-274 (1998)

2. Areces, C., Figueira, D., Figueira, S., Mera, S.: The expressive power of memory logics. Rev. Symb. Log. 2, 290-318 (2011)

3. Areces, C., ten Cate, B.: Hybrid logics. In: Handbook of Modal Logics. Elsevier (2006)

4. Baader, F., Calvanese, D., McGuinness, D.L., Nardi, D., Patel-Schneider, P.F. (eds.): The Description Logic Handbook. Cambridge University Press (2003)

5. Barr, M.: Terminal coalgebras in well-founded set theory. Theoret. Comput. Sci. 114, 299-315 (1993)

6. Börger, E., Grädel, E., Gurevich, Y.: The Classical Decision Problem. Perspectives in Mathematical Logic. Springer, Heidelberg (1997)

7. D'Agostino, G., Visser, A.: Finality regained: A coalgebraic study of Scott-sets and multisets. Arch. Math. Log. 41, 267-298 (2002)

8. Fine, K.: In so many possible worlds. Notre Dame J. Form. Log. 13, 516-520 (1972)

9. Goré, R., Kupke, C., Pattinson, D.: Optimal Tableau Algorithms for Coalgebraic Logics. In: Esparza, J., Majumdar, R. (eds.) TACAS 2010. LNCS, vol. 6015, pp. 114-128. Springer, Heidelberg (2010)

10. Gorín, D., Schröder, L.: Celebrities don't follow their followers: Binding and qualified number restrictions. Technical report, DFKI GmbH (2011)

11. Grädel, E.: On the restraining power of guards. J. Symb. Log. 64 (1999)

12. Horrocks, I., Sattler, U.: A description logic with transitive and inverse roles and role hierarchies. J. Log. Comput. 9, 385-410 (1999)

13. Larsen, K., Skou, A.: Bisimulation through probabilistic testing. Inform. Comput. 94(1), 1-28 (1991)

14. Marx, M.: Narcissists, stepmothers and spies. In: International Workshop on Description Logics, CEUR Workshop Proceedings (2002), CEUR-WS.org

15. Myers, R., Pattinson, D., Schröder, L.: Coalgebraic Hybrid Logic. In: de Alfaro, L. (ed.) FOSSACS 2009. LNCS, vol. 5504, pp. 137-151. Springer, Heidelberg (2009)

16. Pattinson, D.: Coalgebraic modal logic: Soundness, completeness and decidability of local consequence. Theoret. Comput. Sci. 309, 177-193 (2003)

17. Pratt-Hartmann, I.: Complexity of the guarded two-variable fragment with counting quantifiers. J. Log. Comput. 17(1), 133-155 (2007)

18. Schneider, T.: The Complexity of Hybrid Logics over Restricted Classes of Frames. PhD thesis, Univ. of Jena (2007)

19. Schröder, L.: A finite model construction for coalgebraic modal logic. J. Log. Algebr. Prog. 73, 97-110 (2007)

20. Schröder, L., Pattinson, D.: How many toes do I have? Parthood and number restrictions in description logics. In: Principles of Knowledge Representation and Reasoning, KR 2008, pp. 307-218. AAAI Press (2008)

21. Schröder, L., Pattinson, D.: PSPACE bounds for rank-1 modal logics. ACM Trans. Comput. Log. 10, 13:1-13:33 (2009)

22. ten Cate, B., Franceschet, M.: On the Complexity of Hybrid Logics with Binders. In: Ong, L. (ed.) CSL 2005. LNCS, vol. 3634, pp. 339-354. Springer, Heidelberg (2005)

23. van Benthem, J.: Correspondence theory. In: Handbook of Philosophical Logic, vol. 3, pp. 325-408. Kluwer (2001) 